\title{
Environmental and occupational respiratory diseases - 1058. Clinical and diagnostic characteristics of mycoplasma pneumoniae pneumonia in children with lobar pneumonia
}

\author{
Jae Ho Lee ${ }^{1 *}$, Eun Ae Yang ${ }^{1}$, Jin Hwan Kim², Jae Ho Lee ${ }^{1}$ \\ From 2nd WAO International Scientific Conference (WISC 2012) \\ Hyderabad, India. 6-9 December 2012
}

\section{Background}

Mycoplasma pneumoniae (M. pneumoniae) infected lobar pneumonia has increased recently in children in Korea.

\section{Objective}

To evaluate the clinical and laboratory characteristics of lobar pneumonia infected by M. pneumoniae and to find more sensitive diagnostic tool in children.

\section{Methods}

We analyzed medical records of 78 children, admitted to Chungnam National University Hospital and diagnosed with lobar pneumonia by chest X-rays between March 2010 and December 2011. White blood cells, C-reactive protein (CRP), procalcitonin (PCT), specific antibodies to $M$. pneumoniae, and cold agglutinin (CA) were measured at admission. Children were divided into 2 groups: those with M. pneumoniae infection (group A) and those without (group B). Group A children were also subdivided into 2 categories: those with increased CA (group A1) and those without (group A2).

\section{Results}

M. pneumoniae infection usually occurred in summer and autumn. Group A children accounted for $75.6 \%$ $(59 / 78)$ of all cases. The onset age was higher in group A than in group B $(P=0.016)$. WBC counts and PCT values were higher in group $\mathrm{B}$ than in group $\mathrm{A}(P=0.015$ and $P=0.011$ ). Radiologic findings showed that the lower lobe was most commonly involved without predilection for either side and that pleural effusion was present in $13.6 \%$ of all cases. The duration of fever before admission was longer in group A1 than in group A2 $(P=0.019)$.

\section{Conclusions}

The clinical symptoms and signs of lobar pneumonia caused by $M$. pneumoniae infection were more severe and can be accurately diagnosed using serum PCT values than using CRP values.

\section{Author details}

${ }^{1}$ Department of Pediatrics, Chungnam National University School of Medicine, Daejeon, South Korea. ${ }^{2}$ Department of Radiology, Chungnam National University Hospital, Daejeon, South Korea.

Published: 23 April 2013

\section{doi:10.1186/1939-4551-6-S1-P56}

Cite this article as: Lee et al:: Environmental and occupational respiratory diseases -1058 . Clinical and diagnostic characteristics of mycoplasma pneumoniae pneumonia in children with lobar pneumonia. World Allergy Organization Journal 2013 6(Suppl 1):P56. 